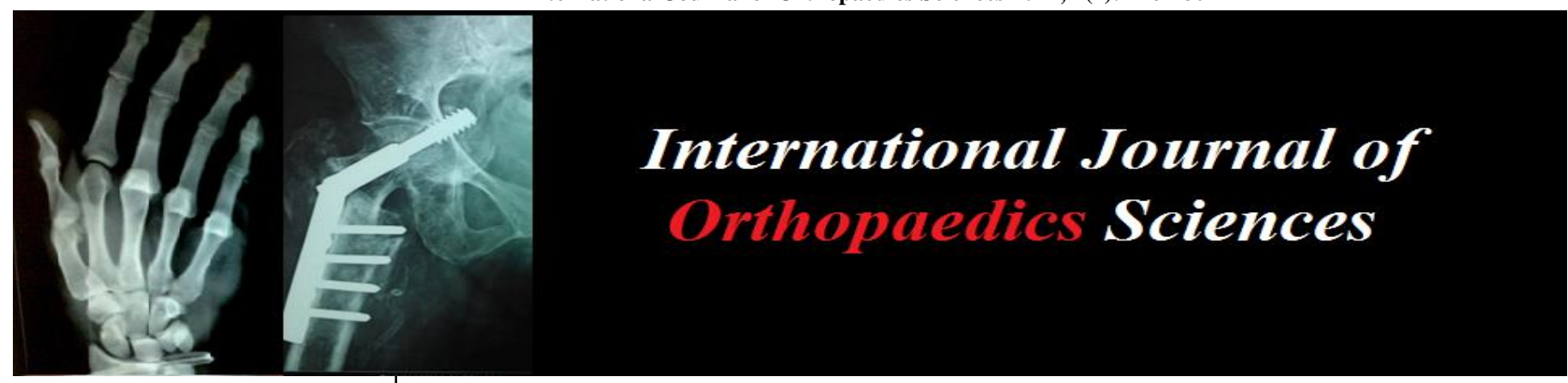

E-ISSN: 2395-1958

P-ISSN: 2706-6630

IJOS 2021; 7(2): 776-780

(C) 2021 IJOS

www.orthopaper.com

Received: 28-02-2021

Accepted: 30-03-2021

Dr. Anson Albert Macwan

3rd Year PG Resident, Department of Orthopaedics, Kalinga Institute of Medical Science Kalinga Institute of Industrial Technology KIIT Road, Patia, Bhubaneswar, Odisha, India

Dr. Saurav Narayan Nanda M.B.B.S, D.N.B (Ortho) Department of Orthopaedic Kalinga Institute of Medical Science, Kalinga Institute of Industrial Technology, KIIT Road, Patia, Bhubaneswar, Odisha, India

Dr. Saswat Samant

M.B.B.S, M.S (Ortho)

Department Of Orthopaedic

Kalinga Institute of Medical

Science Kalinga Institute of

Industrial Technology, KIIT Road,

Patia, Bhubaneswar, Odisha, India

Dr. Ashok Gachhayat

M.B.B.S, D.N.B (Ortho)

Department Of Orthopaedic

Kalinga Institute of Medical

Science Kalinga Institute of

Industrial Technology, KIIT Road,

Patia, Bhubaneswar, Odisha, India

Dr. Govind V J

M.B.B.S, M.S (Ortho)

Department Of Orthopaedic

Kalinga Institute of Medical

Science, Kalinga Institute of

Industrial Technology, KIIT Road,

Patia, Bhubaneswar, Odisha, India

Dr. Anwesit Mohanty

2 rd Year PG Resident

Department Of Orthopaedic

Kalinga Institute of Medical

Science, Kalinga Institute of

Industrial Technology, KIIT Road,

Patia, Bhubaneswar, Odisha, India

Corresponding Author:

Dr. Anson Albert Macwan

3 rd Year PG Resident,

Department Of Orthopaedics,

Kalinga Institute of Medical

Science Kalinga Institute of

Industrial Technology KIIT Road,

Patia, Bhubaneswar, Odisha, India

\section{Standard care of management in orthopaedic patient during COVID 19 pandemic: An institution based guideline}

\author{
Dr. Anson Albert Macwan, Dr. Saurav Narayan Nanda, Dr. Saswat \\ Samant, Dr. Ashok Gachhayat, Dr. Govind V J and Dr. Anwesit Mohanty
}

DOI: $\underline{\text { https://doi.org/10.22271/ortho.2021.v7.i2j.2705 }}$

\begin{abstract}
SARS-CoV 2 pandemic has been spread around the world, the developing countries like India with enormous population, the health care system has been exhausted with patients. Social distancing, using three layered face mask, frequent use of sanitization is helpful preventing measure to spread of these deadly virus. There are no clear cut guidelines available in management of Orthopaedic patients till now. The disease and mortality burden will increase eventually without the effective vaccine and medications. With this ongoing pandemic managing elective orthopaedic surgeries and delayed trauma is a challenge. There should be effective protocol to manage emergency cases with optimum care and formulate standard postoperative rehabilitation strategy to deal with the situation. Orthopaedics training is also affected by this pandemic and that should be taken care of with appropriate measures. These article will help the surgeons and hospitals to formulate proper strategies in managing the patients and to face challenges presented in current pandemic situation. It will guide them to re-establish the orthopaedic care and to face future challenges.
\end{abstract}

Keywords: covid19, orthopedic care, institution guideline, standard care, trauma, hospital care

\section{Introduction}

Coronavirus disease 2019 (COVID-19) originated in the city of Wuhan in Central China, and has spread quickly worldwide. It causes severe acute respiratory syndrome coronavirus 2 (SARS-CoV-2). At present, this newly identified disease is causing a large number of deaths and numerous confirmed cases worldwide and a major life risk to public health ${ }^{[1]}$. In February 2019 covid 19 was declared as public health emergency of international concern ${ }^{[2]}$. The cases started emerging from Europe and then to USA and now almost whole world is affected with it. Presently according to WHO, 162 million cases world-wide with 33 million confirmed deaths and around 222 countries are affected ${ }^{[3]}$. The disease is spreading across the world very quickly and lots of people around the world losing their precious lives.

India is country with population of 1.3 billion, second largest population after china. By the end of July 2019 there were 8 lac cases. Government of India implemented lockdown and compulsory social distancing to fight the disease all over the country to control the disease spread ${ }^{[4]}$. World Health Organization recommended doctor to population ratio of 1:1,000. India is still lacking adequet infrastructure in health sector in both public and private health sectors to fulfil the healthcare need of its growing population ${ }^{[5]}$, and COVID 19 is putting a huge burden on it. The first case of COVID 19 was reported in india on month of January 2020 in southern state of india ${ }^{[6]}$. India was quick to response to the spread of COVID 19 compare to other developed countries like United states, Italy, UK. The "Janata Curfew" was implemented by the prime minister of India in month of march with only 360 active cases. It was the world's largest lockdown of 21 days, which failed to "flatten the curve" and second lockdown was initiated, which further underwent two more extensions ${ }^{[7]}$.

According to the data of health ministry of India on 20 may 2021, the total number of active cases were 26 million with 22 million recoveries. The national recovery rate was $86.74 \%$. Total 20 million tests were done nationwide with mortality rate of $1.11 \%$. 
Currently India has vaccinated over 180 million citizens ${ }^{[15]}$.

\section{Standard Orthopaedic practice during pandemic}

Orthopaedic patients presenting to casualty and outpatient department during COVID 19 pandemic 1. Infection- septic arthritis and osteomyelitis, 2. trauma, 3. elective OA patients with severe symptoms, 4. severe backpain patients, 5. postoperative follow-up patients, 6. post-operative infected cases, 7. metabolic diseases and bone cancer patients.

steps to manage orthopaedic patients:-

\section{Safety precautions}

One of the major hurdle is to segregate COVID 19 infected patients from non-infected patients, doctors as well as staff. To create a safe environment, the patients who are arriving to casualty need to be kept in separate cubicle. Every patient entering the hospital need to be scanned by thermal body temperature check and should be sanitised with hand sanitizer. All patient entering to the hospital should be mandated to wear a mask, preferably 3 layered mask or N95 mask. Attenders accompanying the patients need to be restricted to one attender per person. All the doctors and staff working with patient must be ensure that they are asymptomatic and should be under go regular testing for COVID 19 RTPCR. The examination areas has to be cleaned frequently with $2 \%$ chlorhexidine or $1 \%$ hypochlorite solution including tables, stools, doors and door knobs.

\section{Examination in emergency room}

Doctors and staffs working in casualty need to be equipped with a N95 mask, face shield, hand sanitizers and a pair of gloves. All the patients arriving in the casualty with orthopaedic complaint are mandated to wear compulsory mask during history taking and physical examination. A detailed history of contact with COVID patient and sign and symptoms of COVID infection should be checked in all patients before examination. Compulsory use of PPE by health care provider in emergency department is one of the major protective measure to decrease the spread. All the patients who require admission should undergo screening test of RAPID COVID 19 test and are shifted to isolation ward with a negative report. Those patients are again tested for COVID 19 RTPCR, which takes 24hours on an average. After obtaining a negative RTPCR report, the patients are shifted to Orthopaedic ward and are managed accordingly. The patients with positive COVID report (either ANTIGEN or RTPCR) are directed to COVID hospital and managed accordingly.

\section{Treatment of OPD and elective patients}

All the patients arriving to OPD must be symptom free and need to be checked for temperature. Mask need to be made compulsory for all individuals in OPD area. All the doctors and paramedical staffs should have a face shield. All health care provider should be instructed to follow social distancing and to use hand-sanitizer frequently also to use disposable gloves. All follow up post-operative patients should be segregated and are examined in a single room and instructed for home care if possible to avoid frequent visit. All the elective patients with severe symptoms are examined and documented for their symptoms. Patients with low back pain, avascular necrosis, ankylosing hip, osteoarthritis and rheumatoid arthritis should be prescribed an adequate analgesia to delay and sort of surgical intervention. Bone tumour patients with pathological fracture, infection requiring admission should be investigated with all the routine tests, pre anaesthetic check-up, necessary consultation before operation. Proper history regarding COVID 19 contact, any sign and symptoms and symptomatic people in his family should be documented. RTPCR for COVID 19 for both patient and attendant must be done before admission. All the patient must be educated and stimulated for TELE-Medicine and to use electronic media for queries and follow ups.

\section{Management of trauma cases}

Upon arriving the patient at the emergency room, the triage assessment must be done and all the closed fracture patients should be treated with primary splint and to be tested for COVID 19 rapid antigen. Then, as per protocol they are admitted in Isolation ward. Patients are further tested for RTPCR before definitive management.

All the open fractures, compartment syndrome and suspected vascular injury patients are tested for COVID 19 rapid antigen and all negative patients need to be planned for emergency OT with PPE and all required protection. All the positive patients are shifted to COVID hospital. There should be arrangement of emergency OT in COVID hospital and those COVID positive patients who need any emergency procedure should be managed. Patients requiring fir ICU support should be managed in either COVID ICU or normal ICU as per the COVID report. 


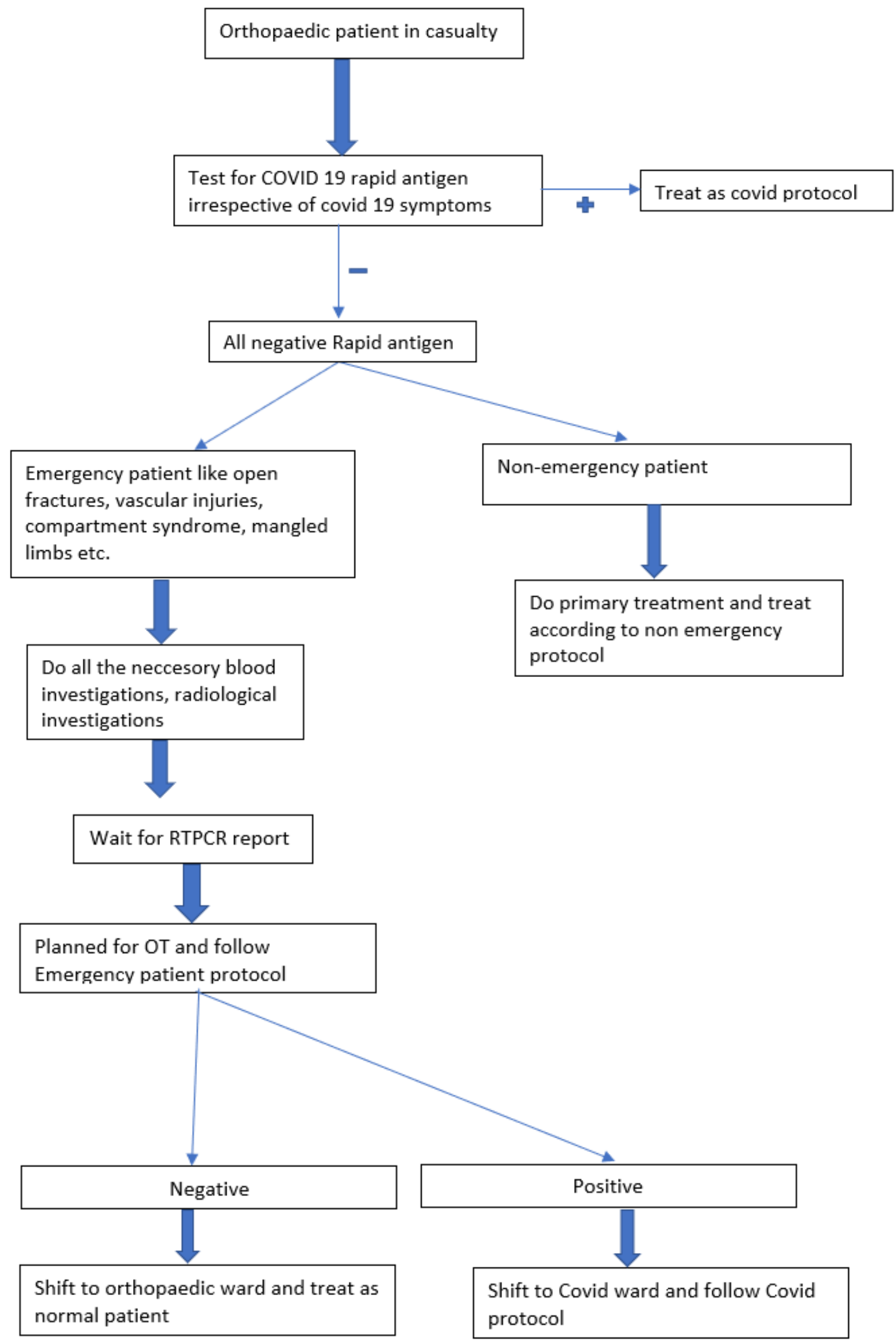

Fig 1: Flow charts for managing patients during pandemic (Fig 1)

\section{Discussion}

COVID 19 has spread to whole world and have saturated the health care system. As so many COVID 19 patients falling sick in very short period of time, the demand of hospital admission is increasing, as well as demand for physicians to treat them has also increased. Along with it increases the demand of PPE Kits, ventilators, ICU care and trained health care workers. Since the beginning of the pandemic there is decrease in the number of orthopaedic patients. There is significant decrease in number of patients coming to OPD, IPD admission, elective surgeries and number of total surgeries. Number of patients presenting to emergency department has also decreased due to lockdown and lless number of RTAs ${ }^{[12]}$.

The decrease in amount of orthopaedic surgeries was seen around the world. There was $44 \%$ reduction in surgeries and 
decease in orthopaedic intervention in upper limb. Lower limb fractures and also replacement surgeries ${ }^{[12]}$. However there was increased in number mortality in proximal femoral fractures in elderly age group. Patralekh et all have done a systematic review in 4355 patients with proximal femoral fractures and reported increased mortality in COVID 19 positive patients and the reason was shown to cytokine storm post operatively [13]

The recent COVID 19 pandemic is expected to continue for coming years. However high patient load compare to healthcare infrastructure is always challenging in India. Since there is decreased in number of elective orthopaedic procedures and musculoskeletal tumour patients. It will eventually lead to increase in orthopaedic related disease burden and also increase in mortality rate. Patients who are RTPCR positive and can be managed conservatively should be treated accordingly with all the precautions taken into consideration, however patient requiring surgeries should wait until asymptomatic and for RTPCR test to become negative. However, we should keep eye on the analgesia, immobilization and neurovascular status time to time.

As the disease spread across the world, the hospitals became of the important zones for treatment and also the potential zones of community transmission. The big issue was how to restart the typical orthopaedic care in middle of pandemic, as it was not only the issue of safety but also as an issue in ethical point of view.[8] The American College of Surgeons (ACS) and the Centers for Medicare \& Medicaid Services (CMS) issued guidelines for elective surgeries. However, the surgeons had difficulties in identifying the elective procedures as there are no clear guidelines and it resulted in confusion ${ }^{[8]}$. Cancelation of all elective procedures created tremendous backlog of patients ${ }^{[9]}$. It is a worldwide challenge to resume the elective procedures during this COVID 19 pandemic. As we know the various stages of epidemic crisis, it is advisable to minimize and differ all the elective surgeries ${ }^{[10]}$. However, the system have to be prepared for gradually restarting of the operative procedures with proper safety of the doctors as well as medical staff in the hospital by the time we reach to normal. As the prevalence of the disease is still high, with limited staff and increasing number of cases which is pressurizing the healthcare system, hospital should act fast and start the essential orthopaedic surgical care while protecting the patient and valuable resources ${ }^{[11]}$

orthopaedic care also need to be resumed as before pandemic, with some changes in treatment protocols and follow-up of the patients. Resuming of the orthopaedic practice should be slow, systematic and strategic. Orthopaedic patients presenting to casualty following trauma due to RTA, fall from standing height in elderly, polytrauma patients, vascular injury patients were managed with proper treatment protocols till the COVID 19 pandemic ends. Patients who are admitted, operated and discharged should try to decrease the infection spread and try to keep themselves safe and healthy. With no clear cut guidelines available for the treatment of the orthopaedic patients, it is difficult to manage them in some part of the country where there are very limited resources available. As now various effective vaccination available, we urge all the patient coming to us get vaccinated and still mandate to follow certain protocols for the smooth work of the orthopaedic care without harming the patient as well as healthcare workers.

Medical education has been always suffered during pandemics. All the medical institutions were shut down following COVID 19 pandemic to decrease the transmission.
The classroom teaching has been shifted to online platforms but the practical teaching including patient centred learning is affected significantly. Academic is severly affected for orthopaedics residents, due to decrease in number of patients as well as number of surgeries. On the other hand, they have to join in the battle against COVID 19 along with other physicians in various COVID hospitals. More stress should be given to improvise and strengthen the online training programme which can able to provide similar training environment like that of physical training programme. The senior post graduates should get more opportunities during this pandemic to get adequate training which can help them in getting employment in future ${ }^{[14]}$.

\section{Conclusion}

Resuming of orthopaedic practice should be safe and strategic for patient care as well as safety of the health care workers. Elective surgeries should be avoided and conservative management should be considered if no superior outcome can be achieved with surgery. Operative time should be minimized as much as possible with minimal surgical interventions. Hospitals and surgeons should follow the advised guideline by national or state health authorities. Medical education also need to be continued using different online platforms. With proper planning and using effective protocols we can help Developing Countries and rest of the world for going back to new normal by modifying the standard treatment. Preventive measures should be taken to preserve infrastructure and manpower to manage ongoing pandemic.

\section{References}

1. Li H, Liu SM, Yu XH, Tang SL, Tang CK. Coronavirus disease 2019 (COVID-19): current status and future perspectives. Int $\mathrm{J}$ Antimicrob Agents. 2020;55(5):105951.

doi:10.1016/j.ijantimicag.2020.105951

2. World Health Organization. Statement on the Second Meeting of the International Health Regulations. Emergency Committee regarding the outbreak of novel coronavirus (2019-nCoV). 2005. https ://www.who.int/news-room/detai 1/30-01-2020-state menton- the-secon dmeet ing-of-the-inter natio nal-healt h-regul ation s-(2005)-emerg ency-commi ttee-regar ding-the-outbr eak-of-novel coron aviru s-(2019-ncov). Accessed 17 Feb 2020.

3. Coronavirus disease

(COVID19)/https://www.who.int/emergencies/diseases/novelcoronavirus-2019/World Health Organization/December 07,2020

4. Ghosh A, Nundy S, Mallick TK. How India is dealing with COVID-19 pandemic. Sensors International. 2020;1:100021. doi:10.1016/j.sintl.2020.100021

5. Kumar R, Pal R. India achieves WHO recommended doctor population ratio: A call for paradigm shift in public health discourse!. J Family Med Prim Care. 2018;7(5):841-844. doi:10.4103/jfmpc.jfmpc_218_18

6. Ministry of Health and Family Welfare/https://www.mohfw.gov.in/MoHFW

7. Venkata-Subramani M, Roman J. The Coronavirus Response in India - World's Largest Lockdown. Am J Med Sci 2020;360(6):742-748. doi:10.1016/j.amjms.2020.08.002

8. Humbyrd CJ, Dunham AM, Xu AL, Rieder TN. Restarting Orthopaedic Care in a Pandemic: Ethical 
Framework and Case Examples. J Am Acad Orthop Surg. 2020. doi: 10.5435/JAAOS-D-20-00871. Epub ahead of print. PMID: 33156215.

9. CovidSurg Collaborative, Nepogodiev D, Bhangu A. Elective surgery cancellations due to the COVID-19 pandemic: global predictive modelling to inform surgical recovery plans. Br J Surg 2020. 12. 10.1002/bjs.11746.

10. Tuech JJ, Gangloff A, Schwarz L. Our challenge is to adapt the organization of our system to the six stages of the epidemic to go beyond the COVID-19 crisis. Br J Surg 2020;10.1002/bjs.11639

11. Neradi D, Hooda A, Shetty A, Kumar D, Salaria AK, Goni V. Management of Orthopaedic Patients During COVID-19 Pandemic in India: A Guide. Indian J Orthop. 2020;54(3):402-407. Published 2020 Apr 27. doi:10.1007/s43465-020-00122-6

12. Kumar S, Shah B, Johari A et al. Covid-19 Pandemic: Resumption of Orthopedic Care and Medical Education. JOIO 2021;55:506-515 https://doi.org/10.1007/s43465-021-00379-5

13. Mohit Kumar Patralekh, Vijay Kumar Jain, Karthikeyan P Iyengar, Gaurav Kumar Upadhyaya, Raju Vaishya. Mortality escalates in patients of proximal femoral fractures with COVID-19: A systematic review and metaanalysis of 35 studies on 4255 patients, Journal of Clinical Orthopaedics and Trauma 2021;18:80-93, https://doi.org/10.1016/j.jcot.2021.03.023.

14. Macdougall C, Dangerfeld P, Katz D, Strain W. The impact of COVID-19 on Medical education and Medical Students. How and when can they return to placements? Med Ed Publish 2020;9(1):159. https://doi.org/10.15694/mep.2020.000159.1.

15. https://pib.gov.in/PressReleasePage.aspx?PRID=1720166 